\title{
AUTOLOGOUS BILAYERED SELF-ASSEMBLED SKIN SUBSTITUTES (SASSS) AS PERMANENT GRAFTS: A CASE SERIES OF 14 SEVERELY BURNED PATIENTS INDICATING CLINICAL EFFECTIVENESS
}

\author{
L. Germain ${ }^{1, *, \$, ~ D . ~ L a r o u c h e ~}{ }^{1}$, B. Nedelec ${ }^{2}$, I. Perreault ${ }^{3,4}$, L. Duranceau ${ }^{3,5}$, P. Bortoluzzi ${ }^{4}$, C. Beaudoin \\ Cloutier $^{1,3}$, H. Genest ${ }^{6}$, L. Caouette-Laberge ${ }^{4}$, A. Dumas $^{6}$, A. Bussière ${ }^{6}$, E. Boghossian ${ }^{3}$, J. Kanevsky $^{7}$, \\ Y. Leclerc ${ }^{6}$, J. Lee ${ }^{7}$, M.T. Nguyen ${ }^{8}$, V. Bernier ${ }^{9}$, B.M. Knoppers ${ }^{8}$, V.J. Moulin ${ }^{1,}$ and F.A. Auger ${ }^{1, \S}$
}

${ }^{1}$ Centre de recherche du CHU de Québec-Université Laval, Regenerative Medicine Division (CRCHU),

Department of Surgery, Faculty of Medicine, Université Laval and Centre de recherche en organogénèse expérimentale de l'Université Laval/LOEX, 1401 18 ième Rue, Quebec, Quebec, G1J 1Z4, Canada

${ }^{2}$ School of Physical and Occupational Therapy, McGill University, Centre de recherche du Centre hospitalier de l'Université de Montréal (CRCHUM), Hôpital de réadaptation Villa Medica,

225 Rue Sherbrooke E, Montreal, Quebec, H2X 1C9, Canada

${ }^{3}$ Université de Montréal, Faculty of Medicine, Department of Surgery, Division of Plastic Surgery, 2900 Boulevard Edouard-Montpetit, Montreal, Quebec, H3T 1J4, Canada

${ }^{4}$ CHU Sainte-Justine, 3175 Chemin de la Côte-Sainte-Catherine, Montreal, Quebec, H3T 1C5, Canada

${ }^{5}$ Unité des Grands-Brûlés, Hôpital Hôtel-Dieu de Montréal, Centre Hospitalier Universitaire de Montréal

(CHUM), 3840 Rue Saint-Urbain, Montreal, Quebec, H2W 1T6, Canada

${ }^{6}$ Centre Hospitalier Universitaire (CHU) de Québec, Université Laval, $140118^{\mathrm{e}}$ Rue,

Quebec, Quebec, G1J 1Z4, Canada

${ }^{7}$ University McGill, Faculty of medicine, Department of Surgery, Division of Plastic Surgery,

3605 Rue de la Montagne, Montreal, Quebec, H3G 2M1, Canada

${ }^{8}$ Centre of Genomics and Policy, McGill University, Faculty of Medicine, Department of Human Genetics,

740 Dr Penfield Avenue, Montreal, Quebec, H3A 0G1, Canada

${ }^{9}$ Department of Molecular Biology, Medical Biochemistry and Pathology, Faculty of Medicine,

Université Laval,1050 Avenue de la Médecine, Quebec, Quebec, G1V 0A6, Canada

$\S$ These authors contributed equally

\begin{abstract}
Split-thickness skin autografts (AGs) are the standard surgical treatment for severe burn injuries. However, the treatment of patients with substantial skin loss is limited by the availability of donor sites for skin harvesting. As an alternative to skin autografts, our research group developed autologous self-assembled skin substitutes (SASSs), allowing the replacement of both dermis and epidermis in a single surgical procedure. The aim of the study was to assess the clinical outcome of the SASSs as a permanent coverage for full-thickness burn wounds. Patients were recruited through the Health Canada's Special Access Program. SASSs were grafted on debrided full-thickness wounds according to similar protocols used for AGs. The graft-take and the persistence of the SASS epithelium over time were evaluated. 14 patients received surgical care with SASSs. The mean percentage of the SASS graft-take was $98 \%$ (standard deviation $=5$ ) at 5 to $7 \mathrm{~d}$ after surgery. SASS integrity persisted over time (average follow-up time: 3.2 years), without noticeable deficiency in epidermal regeneration. Assessment of scar quality (skin elasticity, erythema, thickness) was performed on a subset of patients. Non-homogeneous pigmentation was noticed in several patients. These results indicated that the SASS allowed the successful coverage of full-thickness burns given its high graft-take, aesthetic outcome equivalent to autografting and the promotion of long-term tissue regeneration. When skin donor sites are in short supply, SASSs could be a valuable alternative to treat patients with full-thickness burns covering more than $50 \%$ of their total body surface area.
\end{abstract}

Keywords: Autologous, burn, culture techniques, connective tissue, regenerative medicine, skin, skin grafts, tissue culture, tissue engineering, tissue therapy.

*Address for correspondence: Lucie Germain, CHU de Québec-Université Laval, LOEX, Aile-R, $140118^{\text {ième }}$ Rue, Quebec, Quebec, G1J 1Z4, Canada.

Telephone number: +1 $4185254444 \quad$ Email: lucie.germain@fmed.ulaval.ca

Copyright policy: This article is distributed in accordance with Creative Commons Attribution Licence (http://creativecommons.org/licenses/by-sa/4.0/). 


\section{Introduction}

Large full-thickness burns continue to be a surgical challenge. The standard treatment for deep-partial or full-thickness injuries is the application of splitthickness skin autografts (AGs) harvested from an uninjured skin donor site. However, insufficient or unavailable donor sites for harvesting can occur in patients injured by burns over a large percentage of their total body surface area (TBSA). In such instances, surgeons must harvest the same donor site several times, with delays in treatment, as donor sites need time to heal prior to re-harvesting. Notably, these multiple harvests can ultimately lead to donor site exhaustion, especially in fragile patients. Furthermore, multiple donor site harvests are associated with morbidity and can cause pain, fluid loss, infection, prolonged time of healing, hypertrophic scarring and undesirable pigmentation (Fowler and Dempsey, 1998; Voineskos et al., 2009). Treatment strategies developed to overcome the limited availability of donor sites are, for instance, the regenerative dermal template, the wide meshed skin autografts and the Meek technique (Zuo et al., 2017). However, these approaches have major drawbacks, such as the necessity to reconstruct the epidermal layer from limited donor sites and scarring associated with Meek and wide meshed skin autografts.

Significant progress has been made over the past 25-years in the development of tissue-engineered bilayered skin substitutes. A few models tested on humans to treat acute full-thickness burn wounds are known (Boyce et al., 1995a; Boyce et al., 1999; Boyce et al., 2002; Boyce et al., 2006; Boyce et al., 2017; Golinski et al., 2014; Gomez et al., 2011; Harriger et al., 1995; Kuroyanagi et al., 1993; Llames et al., 2004; Llames et al., 2006; Sheridan et al., 2001; Takami et al., 2014). The most studied cultured skin substitute consists of collagen-glycosaminoglycan substrate populated with autologous fibroblasts and keratinocytes [also known as engineered skin substitute (ESS)]. This model, tested in over 125 patients, shows promising clinical results (Boyce et al., 1995a; Boyce et al., 1999; Boyce et al., 2002; Boyce et al., 2006; Boyce et al., 2017; Harriger et al., 1995). However, successful engraftment of this skin substitute requires special wound bed preparation and specific post-operative wound care (Boyce et al., 1995a; Boyce et al., 1995b; Boyce et al., 2006). Three other studies also report the clinical results of a tissueengineered skin substitute composed of autologous keratinocytes and fibroblasts, seeded onto a matrix elaborated from human plasma and produced at a relatively low cost. Current results support its use as an adjunct treatment for large burns. However, this skin substitute model is vulnerable to infection, which significantly lowers its percentage of grafttake (Gomez et al., 2011; Llames et al., 2004; Llames et al., 2006). Other studies report the use of different skin substitutes tested on burned areas smaller than $180 \mathrm{~cm}^{2}$ in a small number (seven or fewer) of patients with acute burns (Golinski et al., 2014; Kuroyanagi et al., 1993; Sheridan et al., 2001; Takami et al., 2014), limiting the ability to draw definitive conclusions.

The self-assembled skin substitute (SASS) is an autologous tissue-engineered skin substitute that allows the replacement of both dermis and epidermis in a single surgical procedure (Larouche et al., 2016; Michel et al., 1999). The SASS dermis is composed of a collagen-rich extracellular matrix secreted by the patient's fibroblasts (Pouliot et al., 2002). The overlying stratified epidermis contains all the layers of normal human epidermis, including the protective stratum corneum (Lavoie et al., 2013). In mouse models, epithelial stem cells persist in the SASS after production and regenerate the epidermis after grafting (Lavoie et al., 2013; Pouliot et al., 2002). A successful clinical trial shows treatment of six patients with venous ulcers using SASSs (Boa et al., 2013).

The present study reported the first case series of patients suffering from severe full-thickness skin loss, who were treated during the acute phase with SASSs for permanent coverage of their full-thickness wounds. Success of the procedure was evaluated based on the presence of a stable epithelium over time and scarring comparable to that of AGs. Furthermore, elasticity, erythema, melanin-content-related brown colorations and thickness of the SASSs were measured post-grafting and compared with AGs and uninjured skin in a subgroup of patients.

\section{Materials and Methods}

\section{Population}

This study was a case series, which followed 17 severely burned patients enrolled by their physician between August 2005 and October 2014 through the Special Access Program (SAP) of Health Canada. These patients received treatment with autologous SASSs produced at the Centre de recherche du CHU de Québec-Université Laval. In Canada, the SAP is designed to provide access to non-marketed drugs or health products for patients with extremely serious or life-threatening conditions who require emergency and/or compassionate therapies, when other treatments/therapies have failed, are unsuitable or are not available. Proper informed consent for the SASS treatment was obtained from all patients, as required by institutional policies and guidelines.

A research project was conducted to measure the skin characteristics of patients treated with the SASSs. This project was approved by the McGill University Institutional Review Board, the CHUM scientific and ethics committee, the ethics committee of Villa Medica Rehabilitation Hospital and the ethics committee of CHU de Québec-Université Laval. The evaluation was conducted at Villa Medica Rehabilitation Hospital and CHU de Québec-Université Laval.

The patients, two of which were paediatric burn victims, were treated in the burn units of three different hospitals in the Quebec province: the 
CHUM, the CHU Sainte-Justine and the CHU de Québec-Université Laval. Upon arrival at the burn unit and until the SASSs were available, the medical team followed the course of healing of the patients and administered standard care, including grafting surgeries using AGs. Once available, the SASSs were used on uncovered full-thickness wounds.

\section{Cell isolation and culture}

For each patient, a skin sample ranging between 2.3 to $10 \mathrm{~cm}^{2}$ [mean $5.8 \mathrm{~cm}^{2}$, standard deviation $\left.(\mathrm{SD})=2.7 \mathrm{~cm}^{2}, n=14\right]$ was harvested as early as possible after injury, usually during the first week post-trauma (mean $4.9 \mathrm{~d}, \mathrm{SD}=8.6 \mathrm{~d}$ ). Each skin biopsy was put into a sterile container filled with cold $\left(4{ }^{\circ} \mathrm{C}\right)$ transport medium [90 \% Dulbecco-Vogt modified Eagle medium (Corning), $10 \%$ foetal bovine serum (Seradigm, Providence, UT, USA), $100 \mathrm{UI} / \mathrm{mL}$ penicillin G (Fresenius Kabi Canada Ltd, Richmond Hill, ON, Canada), $25 \mu \mathrm{g} / \mathrm{mL}$ gentamicin (Galenova Inc., St-Hyacinthe, QC, Canada), $0.5 \mu \mathrm{g} /$ $\mathrm{mL}$ amphotericin B (Bristol-Myers Squibb)] and transferred to the LOEX cell culture facility. Keratinocytes and fibroblasts were extracted and cultured as described (Bisson et al., 2013; Larouche et al., 2009; Lavoie et al., 2013). Dermal fibroblasts were cultured in fibroblast medium (DulbeccoVogt modified Eagle medium supplemented with $10 \%$ foetal bovine serum, $100 \mathrm{U} / \mathrm{mL}$ penicillin and $25 \mu \mathrm{g} / \mathrm{mL}$ gentamicin) until $100 \%$ confluence was reached (8-12 d, mean $9.6 \mathrm{~d}, \mathrm{SD}=1.1 \mathrm{~d}, n=14)$. Then, they were detached using trypsin and cultured for another passage (6-8 d, mean $6.9 \mathrm{~d}, \mathrm{SD}=0.6 \mathrm{~d}, n=14$ ). Fibroblasts were either seeded to produce dermal substitutes (see "Skin tissue production" section) or cryopreserved. For tissue production, fibroblasts were used at passages two to six. Keratinocytes were grown on a feeder layer of irradiated human fibroblasts and cultured in keratinocyte medium [Dulbecco-Vogt modified Eagle medium: Ham's F12, ratio 3 : 1, $24.3 \mu \mathrm{g} / \mathrm{mL}$ adenine (Corning), $5 \mu \mathrm{g} / \mathrm{mL}$ insulin (Sigma-Aldrich), $0.4 \mu \mathrm{g} / \mathrm{mL}$ hydrocortisone (Teva Canada Ltd., Scarborough, ON, Canada), $0.212 \mu \mathrm{g} /$ $\mathrm{mL}$ isoproterenol hydrochloride (Sandoz Canada, Boucherville, QC, Canada), 5 \% bovine HyClone FetalClone II serum (GE Healthcare), $10 \mathrm{ng} / \mathrm{mL}$ human epidermal growth factor (Austral biologicals, San Ramon, CA, USA), $100 \mathrm{U} / \mathrm{mL}$ penicillin and 25 mg/ $\mathrm{mL}$ gentamicin]. Keratinocytes were detached using trypsin before becoming confluent and subcultured for one passage. After 5 to $8 \mathrm{~d}$ (mean $6.8 \mathrm{~d}, \mathrm{SD}=1.0 \mathrm{~d}$, $n=14)$, keratinocytes were cryopreserved. For tissue production, keratinocytes were thawed, subcultured for one passage, trypsinised and counted using a cell counter (Beckman Coulter ${ }^{\circledR}$ Life Sciences,) before seeding. Therefore, they were seeded at the third passage onto the reconstructed dermis.

\section{Skin tissue production}

SASSs were produced as described (Gauvin et al., 2013; Larouche et al., 2009; Larouche et al., 2016). Patients' fibroblasts were seeded at a density of $4 \times 10^{3}$ cells/ $\mathrm{cm}^{2}$ and cultured in fibroblast medium containing $50 \mu \mathrm{g} / \mathrm{mL}$ ascorbic acid (Galenova Inc.) until the formation of a fibroblast-derived tissue sheet. After 14 to $29 \mathrm{~d}$ (mean $21.6 \mathrm{~d}, \mathrm{SD}=4.0 \mathrm{~d}, n=14$ ), three of these dermal tissues were stacked to form a reconstructed dermis. Then, keratinocytes were seeded onto the reconstructed dermis. From 2005 to 2014, the method has evolved over time and the seeding density was decreased from $2.5 \times 10^{5}$ to $1.0 \times 10^{5}$ keratinocytes $/ \mathrm{cm}^{2}$ (mean $2.1 \times 10^{5}, \mathrm{SD}=0.4 \times 10^{5}$ keratinocytes $\left./ \mathrm{cm}^{2}\right)$. The tissue construct was cultured in keratinocyte medium containing $50 \mu \mathrm{g} / \mathrm{mL}$ ascorbic acid for 4 to $7 \mathrm{~d}$ (mean $6.5 \mathrm{~d}, \mathrm{SD}=0.9 \mathrm{~d}, n=14$ ). Next, the construct was transferred on a support, to maintain the tissue at the air-liquid interface, and further cultured for 9 to $14 \mathrm{~d}$ (mean $11.2 \mathrm{~d}, \mathrm{SD}=1.6 \mathrm{~d}, n=14$ ) in keratinocyte medium exempt of epidermal growth factor and containing $50 \mu \mathrm{g} / \mathrm{mL}$ ascorbic acid. The production time of the SASSs was 39 to $54 \mathrm{~d}$ (mean $46.2 \mathrm{~d}$, $\mathrm{SD}=4.5 \mathrm{~d}, n=14)$. The final product was tested for the presence of aerobic, obligate anaerobic and microaerophilic microorganisms with thioglycolate broth and all SASSs were negative prior to grafting.

Ready-to-graftSASSs comprised of an ADAPTIC ${ }^{\mathrm{TM}}$ non-adhering dressing (Acelity, San Antonio, TX, USA) fixed on its upper surface with a LIGACLIP ${ }^{\circledR}$ (Ethicon Endo-Surgery, Guaynabo, Puerto-Rico) to facilitate transport and manipulation during the skin graft surgery. Each SASS was shipped on a transport agar gel composed of Dulbecco's modified Eagle medium, $0.7 \%$ agarose (J.T. Baker, Phillipsburg, MT, USA), $100 \mathrm{UI} / \mathrm{mL}$ penicillin $\mathrm{G}$ and $25 \mu \mathrm{g} / \mathrm{mL}$ gentamicin.

\section{Tissue analysis}

Reference samples of all SASS batches were collected before grafting and processed subsequently for histological analysis. For the first patient, one $3 \mathrm{~mm}$ punch biopsy of a SASS-grafted site was obtained at 3, 6 and 10 weeks, as well as 6 and 20 months post-grafting and processed for histological and immunofluorescence analyses.

For the histological analysis of the SASS reference samples and post-grafted SASS biopsies, tissues were fixed overnight in HistoChoice ${ }^{\circledR}$ (Amresco, Solon, $\mathrm{OH}, \mathrm{USA}$ ) and embedded in paraffin. $5 \mu \mathrm{m}$-thick sections were coloured with Masson's trichrome (using Weigert's haematoxylin, fuchsinponceau and aniline blue) or with haematoxylin eosin saffron. A normal human skin specimen from the LOEX biological material bank, previously approved by the institutional ethics committee for utilisation in research, was used as control. For immunofluorescence analysis, unfixed SASS reference samples and post-grafted SASS biopsies were embedded in Tissue-Tek ${ }^{\circledR}$ OCT Compound (Sakura Finetek, Torrance, CA, USA) and frozen in liquid nitrogen. Immunofluorescence staining on frozen specimens was performed as described previously (Larouche et al., 2005). Sections were 
permeabilised with acetone $\left(10 \mathrm{~min}\right.$ at $\left.-20{ }^{\circ} \mathrm{C}\right)$ before labelling with mouse anti-CD49f (clone 450-30A, recognises the $\alpha 6$ subunit of integrin, BioRad), mouse anti-human keratin 19 (clone A53-B/ A227, gift from U. Karsten, Institute of Biological Sciences, University of Rostock, Germany), mouse anti-PECAM-1 (clone P2B1, EMD Millipore), mouse anti- $\alpha 3$ subunit of integrin (clone HB-8530, VM2, ATCC, Manassas, VA, USA), rabbit anti-type VII collagen (234192, EMD Millipore) and rabbit antihuman type IV collagen (gift from J.A. Grimaud, Pasteur Institute, Lyon, France). Cell nuclei were counterstained with Hoechst reagent 33258 (Sigma Chemical). For transmission electron microscopy analysis, samples were fixed overnight with $2.5 \%$ glutaraldehyde (Canemco Inc., Gore, QC, Canada), washed with $0.1 \mathrm{M}$ cacodylate buffer (Mecalab Ltd., Montreal, QC, Canada) and post-fixed with $1 \% \mathrm{OsO}_{4}$ for $90 \mathrm{~min}$. Samples were dehydrated in a graded concentration of ethanol, embedded in Epon (Poly/ Bed $^{\circledR}$ 812, Polysciences, Inc., Warrington, PA, USA), cut into thin sections and stained with uranyl acetate and lead citrate, as described previously (Black et al., 1998).

\section{SASS thickness measurement before grafting}

The SASS thickness was measured using histological slide images (magnification: 20×) of SASS reference samples stained with Masson's trichrome. For data acquisition, an AxioImager microscope, coupled with AxioCam ICc1 controlled by Axio-Vision 4.8.2 software, was used. Measures were taken on a minimum of six SASS reference samples per patient.

\section{Treatment}

All patients were admitted to intensive burn care units and received care from a multidisciplinary team of highly trained experts. The burn depth and extent was estimated based on clinical evaluations performed by an experienced burn plastic surgeon. Treated patients had full-thickness burns over more than half of their TBSA. After excision of burned necrotic tissues (Fig. 1), wounds were covered with skin allografts until AGs or SASSs were available.
Cultured autologous epidermis (CAE) was used as specialised dressing on donor sites to accelerate their healing. SASSs were grafted following a similar protocol to AG use. The handling and grafting of SASSs were similar to that of AGs and no significant pitfalls or problems were encountered. Briefly, after surgical debridement of allografts down to healthy tissue, haemostasis was completed and SASSs were applied to the wound bed and secured with surgical Histoacryl ${ }^{\circledR}$ glue (TissueSeal, Ann Arbor, MI, USA), staples or a mix of both. At this point (more than six weeks after arrival in the burn unit), wounds were considered as being full-thickness by the burn plastic surgeon based on clinical evaluations. For each patient, all the anatomical sites grafted with the SASSs were photographed and drawn accordingly on a body chart. The post-operative care was the same for AGs and SASSs. However, the clinical practice has evolved over time and one of the following three protocols was used simultaneously on AGs and SASSs, depending on the study period: Jelonet ${ }^{\mathrm{TM}}$, Bactigras $^{\mathrm{TM}}$ or Acticoat ${ }^{\mathrm{TM}}$ (Smith \& Nephew), followed by a conventional bolster type of dressing. Grafts applied on limbs were splinted after dressing was completed. Dressings were removed to evaluate graft-take between post-operative day 5 to 7 .

\section{Follow-up and outcome assessment}

The percentage TBSA burned was estimated using the Wallace "Rules of Nines" for adults and the Lund and Browder Chart for paediatric patients (Kyle and Wallace, 1950; Lund and Browder, 1944). Graft-take was evaluated by a surgeon and was defined as the percentage of the treated area that was re-epithelialised at post-operative day 5 to 14 . The period for SASS epithelial survival started at the date of the first treatment with the SASSs and continued until the date of the last evaluation of the patient made by a burn plastic surgeon. Epithelial cell survival was estimated based on the absence of skin breakdown and on the presence of a completely epithelialised dry skin surface at the anatomic sites that received the SASSs, as noted on the body chart (the gluteal region was excluded from the analyses).
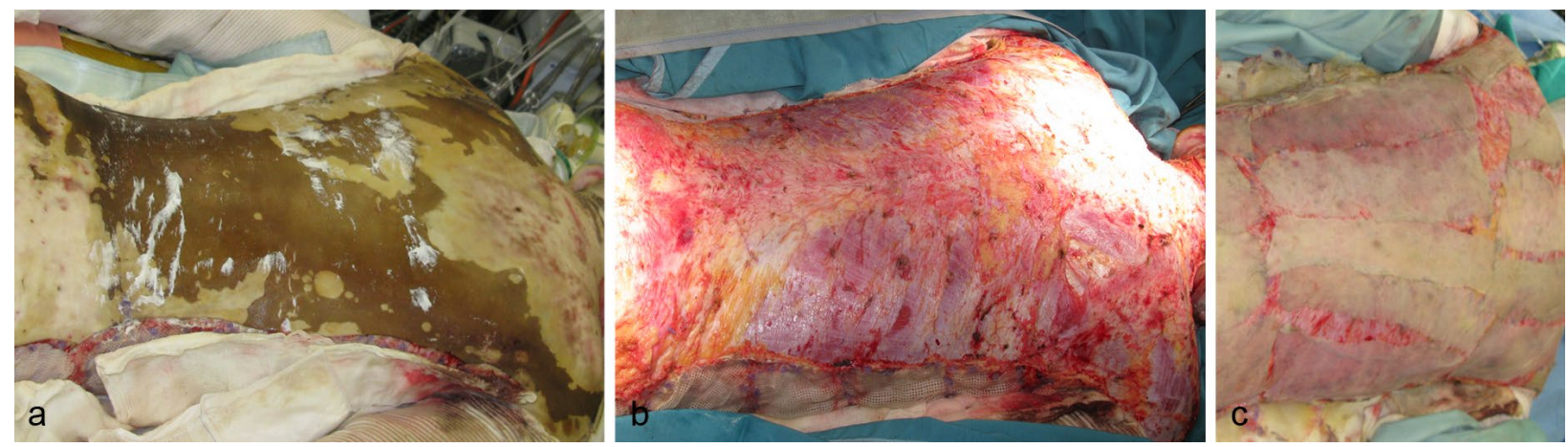

Fig. 1. Full-thickness burn injury before and after excision, and after application of allografts. (a) Representative picture of a full-thickness burn injury on the back of a Caucasian patient $4 \mathrm{~d}$ following his admission at the burn unit. (b) After excision of burned necrotic tissues, (c) the wound was temporarily covered with skin allografts until SASSs were available. 
Patient demographics, complications, hospitalisationrelated data and surgical details were noted throughout the follow-up. Baux score was defined as the sum of the age in years and the percentage of TBSA burned (Dokter et al., 2014). Revised Baux and predicted mortality were calculated as described previously (Osler et al., 2010).

\section{Measurement of skin characteristics}

7 patients who received the SASSs underwent treatments in Villa Medica Rehabilitation Hospital and CHU de Québec-Université Laval and all patients (mean age post-burn $41, \mathrm{SD}=15$, range 17-64 years, $n=7$ ) signed a written consent to participate in the project. The measurement of skin characteristics was performed as described previously (Nedelec et al., 2016). SASSs characteristics such as elasticity, erythema, melanin and thickness were evaluated and compared to AGs at a site free from hypertrophic scar. Cutometer ${ }^{\circledR}$ dual MPA 580 (Courage + Khazaka electronic $\mathrm{GmbH}$, Cologne, Germany) was used to measure maximal skin pliability. A Mexameter ${ }^{\circledR}$ MX18 (Courage + Khazaka Electronic $\mathrm{GmbH}$ ) was used to measure erythema (vascularity) and melanin index. A high frequency ultrasound DermaScan C (Cortex Technology, Hadsund, Denmark) was used to measure skin thickness, as previously described (Nedelec et al., 2008; Nedelec et al., 2016). For three patients, uninjured skin area was also measured. For each evaluation, the most representative sites of the treated area were chosen.

\section{Statistical analysis}

Mean differences of patient-matched skin characteristics (thickness, elasticity, erythema and melanin content) between SASSs and AGs or between SASSs and uninjured skin were compared with the bilateral Wilcoxon signed-rank test. The statistically significant threshold was set at $p<0.05$.

\section{Results}

Between August 2005 and October 2014, 17 requests were submitted to Health Canada through the SAP to use autologous SASSs to treat severely burned patients with a life-threatening condition. All requests were granted. Fig. 2 shows the profile of the clinical case series. Two male patients died before SASS grafting (22, $29 \mathrm{~d}$ post-burn) and one male patient was not treated with SASS as his wounds were covered with AGs before SASSs could be available.

SASSs were successfully produced from all biopsies. From the initial biopsy, the production delay ranged from 56 to $71 \mathrm{~d}$ (mean $62.7 \mathrm{~d}, \mathrm{SD}=4.8 \mathrm{~d}$, $n=14)$. SASS thickness before grafting varied from 127 to $286 \mu \mathrm{m}$ (mean $187 \mu \mathrm{m}, \mathrm{SD}=43 \mu \mathrm{m}, n=14$ ). All SASSs produced were resistant to handling and accordingly tailored to fit irregular wound surfaces.

Fig. 3a shows macroscopic characteristics of the SASS before surgery. For the first treated patient, histological analysis and immunostainings were performed to evaluate the integrity and the persistence of stem cells within the SASS before and after surgery. Histological analyses revealed the presence of a tissue comparable in structure with normal human skin (Fig. 3, compare b with $\mathbf{c}$ and $\mathbf{d}$ ) although rete ridges and skin appendages were absent. A small subset of basal keratinocytes expressing K19 was present before surgery and was also detected $21 \mathrm{~d}$ after grafting (Fig. 3e,f, arrowheads). A continuous labelling of the $\alpha 6$ subunit of integrin and type VII collagen was detected at the dermo-epidermal junction before and after grafting (Fig. 3g-j), indicating a continuous basement membrane, the structure responsible for the adhesion of the epidermis to the dermis. Immunofluorescence analysis of platelet and endothelial cell adhesion molecule (PECAM) and type IV collagen confirmed the presence of blood vessels throughout the SASS dermis (Fig. 3k, arrows). Ultrastructural analysis showed typical structures of the basement membrane at the dermo-epidermal junction, such as lamina densa, lamina lucida and hemidesmosomes (Fig. 31), as well as densely organised collagen fibres within the SASS dermis (Fig. 3m).

SASSs were implanted by 11 different surgeons in 14 patients with full-thickness burn wounds of different aetiologies - fire/flame, scald and flash

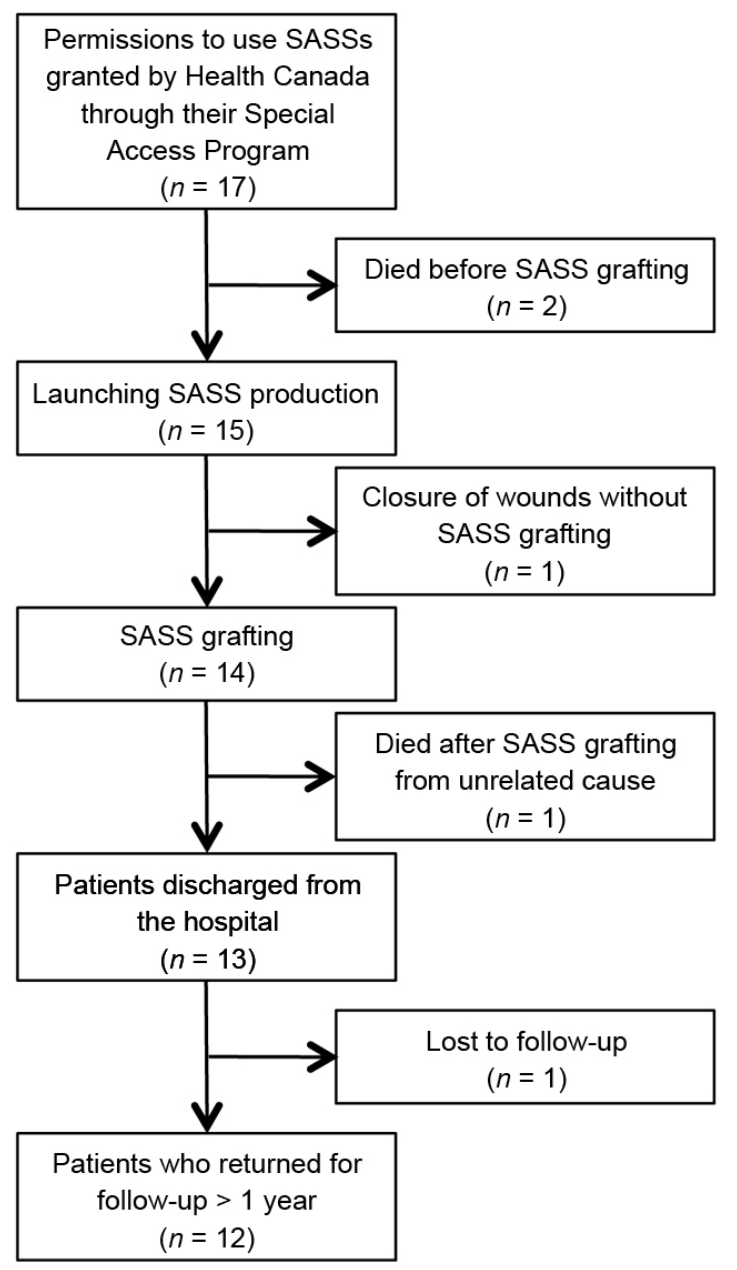

Fig. 2. Profile of the clinical case series. 

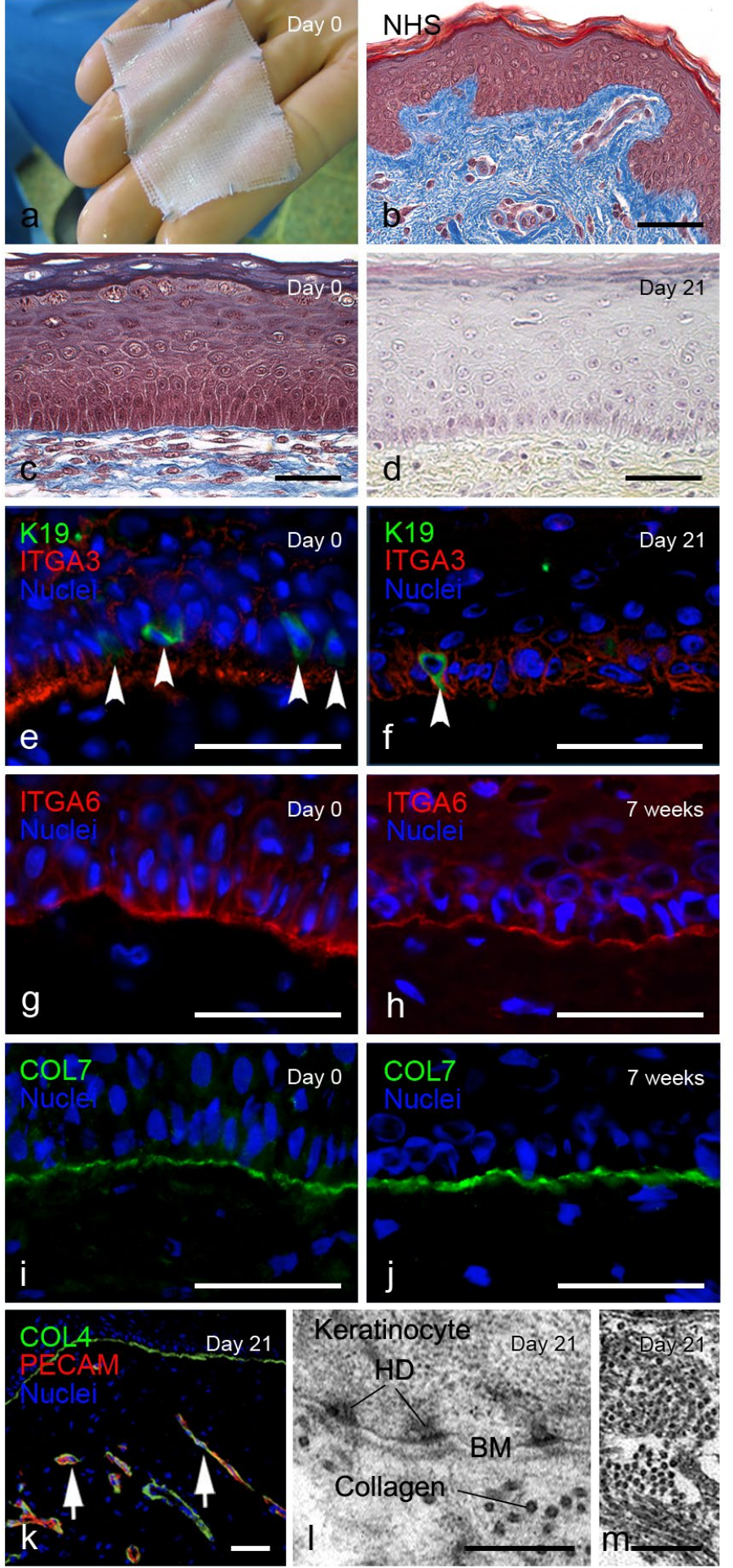

(Table 1) - using techniques similar to AGs. Table 1 shows the characteristics of the population. Successful engraftment was achieved in all patients with no infectious complications or graft loss. The graft-take was estimated at $98 \%(\mathrm{SD}=5 \%)$ at the analysed sites (lower limbs, upper limbs, back, abdominal region or thorax). One female patient died due to infectious complications unrelated to the SASS treatment (191 d post-burn).

Among survivors, all patients underwent multiple skin graft surgeries ranging from 11 to 23 major surgeries. The average number of surgeries involving
Fig. 3. SASSs before and after grafting on a burn patient. (a) Macroscopic aspect of the in-vitro-cultured SASS before grafting. (b-d) Histological cross-sections stained with $(\mathbf{b}, \mathbf{c})$ Masson's trichrome or (d) haematoxylin eosin saffron, showing tissue organisation of the SASS (c) before grafting, which is comparable to (b) normal human skin - although rete ridges, capillaries and appendages were absent - (d) $21 \mathrm{~d}$ after grafting. Note that the histological pictures are representative of the SASS reference sample analysed for each SASS batch. (ek) SASS sections immunolabeled for $(\mathbf{e}, \mathbf{f})$ the stem-cell-associated keratin 19 (green, arrowheads), (e,f) the $\alpha 3$ subunit of integrin (red), (g,h) the $\alpha 6$ subunit of integrin (red), $(\mathbf{i}, \mathbf{j})$ the type VII collagen (green), (k) the type IV collagen (green) and the platelet endothelial cell adhesion molecule (red), $(\mathbf{e}, \mathbf{g}, \mathbf{i})$ before and $(\mathbf{f}, \mathbf{h}, \mathbf{j}, \mathbf{k})$ after grafting. Cell nuclei were stained in blue with Hoechst. (k) $21 \mathrm{~d}$ after grafting, the SASS was vascularised (arrows). (1,m) Representative transmission electron microscopy results of the SASS showing (1) the dermo-epidermal junction and $(\mathbf{m})$ dermal region $21 \mathrm{~d}$ after grafting. $\mathrm{BM}$, basement membrane; COL4, type IV collagen; COL7, type VII collagen; HD, hemidesmosomes, ITGA3, $\alpha 3$ subunit of integrin; ITGA6, $\alpha 6$ subunit of integrin; K19, keratin 19; NHS, normal human skin; PECAM, platelet endothelial cell adhesion molecule. Scale bars: $\mathbf{b}-\mathbf{k}=50 \mu \mathrm{m}$; $\mathbf{l}, \mathbf{m}=500 \mathrm{~nm}$.
AGs, allografts and SASSs is described in Table 1. CAE was used as a living dressing to accelerate the healing of donor sites. Complete depletion of donor sites was observed in two paediatric patients that received the SASSs on $49 \%$ and $53 \%$ of their burn surface area. The last skin graft surgery took place from 66 to $171 \mathrm{~d}$ post-burn. All patients were on mechanical ventilation for more than $10 \mathrm{~d}$ and developed complications unrelated to the SASSs. The most frequent was pneumonia (Table 1). In six patients, partial or complete AG loss was observed, following at least one surgery. 
Table 1. Characteristics of the population treated with SASSs. ICU-LOS, length of stay in the intensive care unit. ${ }^{1}$ Among patients treated with SASSs. ${ }^{2}$ Among survivors. ${ }^{3} \mathrm{CAE}$ was used as a living dressing to help donor sites to heal faster. ${ }^{4}$ Baux score: defined as the sum of age in years and percent body burned. ${ }^{5}$ Revised Baux score: defined as the sum of age in years and percent body burned $+17^{*}\left({ }^{*}\right.$ inhalation injury, yes $=1$, no $=0$ ). ${ }^{6}$ Calculation of the predicted probability of death from the logistic regression model based on the revised Baux score (Osler et al., 2010).

\begin{tabular}{|c|c|c|c|c|c|}
\hline Parameters & $n(\%)$ & Mean (SD) & Median & Min & Max \\
\hline Age (years) & 14 & $34(16)$ & 38 & 10 & 63 \\
\hline Male/female & $12 / 2$ & & & & \\
\hline Caucasian/other & $13 / 1$ & & & & \\
\hline TBSA burn (\%) & 14 & $74(13)$ & 74 & 52 & 92 \\
\hline Initial biopsy area $\left(\mathrm{cm}^{2}\right)$ & 14 & $5.8(2.7)$ & 5.4 & 2.3 & 10 \\
\hline Grafted SASS area $\left(\mathrm{cm}^{2}\right)$ & 14 & $2509(1835)$ & 1996 & 420 & 6295 \\
\hline Burn area grafted with SASS (\%) & 14 & $19(15)$ & 14 & 3 & 53 \\
\hline \multicolumn{6}{|l|}{ Causes $^{1}$} \\
\hline Fire/scald/flash & $12 / 1 / 1$ & & & & \\
\hline SASS graft-take ${ }^{1}(\%)$ & 14 & $98(5)$ & 100 & 85 & 100 \\
\hline 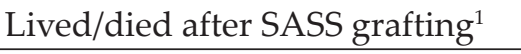 & $13 / 1$ & & & & \\
\hline Number of graft surgeries ${ }^{2}$ & 13 & $16(4)$ & 17 & 11 & 23 \\
\hline AG & 13 & $7(2)$ & 7 & 4 & 10 \\
\hline Allografts & 13 & $9(4)$ & 9 & 3 & 17 \\
\hline SASS & 13 & $3(2)$ & 3 & 1 & 9 \\
\hline Last skin graft surgery $(\mathrm{d})^{2}$ & 13 & $113(31)$ & 113 & 66 & 171 \\
\hline CAE applied on donor sites ${ }^{3}$ & 13 & $6(2)$ & 6 & 2 & 10 \\
\hline ICU-LOS $(\mathrm{d})^{2}$ & 13 & $135(43)$ & 137 & 74 & 240 \\
\hline Ventilation length $(\mathrm{d})^{2}$ & 13 & $64(40)$ & 60 & 11 & 149 \\
\hline Pneumonia $^{2}$ & $12(92.3)$ & & & & \\
\hline Autograft loss ${ }^{2}$ & $6(46.2)$ & & & & \\
\hline SASS loss ${ }^{2}$ & $0(0)$ & & & & \\
\hline Reconstructive surgery $^{2}$ & $9(69.2)$ & & & & \\
\hline Baux score ${ }^{2,4}$ & 13 & $108(19)$ & 106 & 78 & 143 \\
\hline Inhalation/no inhalation injury ${ }^{2}$ & $7 / 6$ & & & & \\
\hline Revised Baux score ${ }^{2,5}$ & 13 & $116(20)$ & 119 & 80 & 160 \\
\hline Predicted mortality ${ }^{2,6}$ & 13 & $52.7(26.9)$ & 60 & 6.8 & 97.3 \\
\hline
\end{tabular}

The Baux score is the sum of age and burn size and is associated to the probability of death of burn victims (Dokter et al., 2014). For a given Baux score, patients with an inhalation injury are at higher risk of mortality than those without. For the 13 survivors treated with the SASSs, the mean Baux score was 108 $(\mathrm{SD}=19)$ and 7 of them had an inhalation injury. Using the revised Baux score, which considers the effect of an inhalation injury, the initial predicted probability of death for these patients was calculated to range from 6.8 to $97.3 \%$, with an average of $52.7 \%$ $(\mathrm{SD}=26.9 \%)$ and a median value of $60.0 \%$ (Osler et al., 2010).

Epithelial cell survival after grafting is illustrated in Fig. 4, based on the last follow-up date since SASS application. No loss of the epithelium was observed during the first year post-intervention or reported subsequently among the 12 patients who returned for follow-up (average follow-up time: 3.2 years, $\mathrm{SD}=1.9$ years). SASS survival for up to 8.4 years was observed.

\section{Macroscopic aspects of the SASSs after grafting}

5 representative cases of patients treated with SASSs are shown in Fig. 5-9. Typically, 2 weeks after grafting, SASS-grafted sites showed adherent and uniform skin-like tissue. The epithelium tightly adhered to the connective tissue without visible blistering or ulceration. The healed SASS-grafted sites were stable, pliable, smooth and generally hypopigmented. Neither hair nor sweating were observed at SASS-grafted sites. Usually, the junctions between the juxtaposed SASSs remained slightly discernible over time (Fig. 5f,h, Fig. 6k and Fig. 7i, arrows). 


\section{Elasticity and thickness of the SASSs after grafting}

In all cases, SASS-grafted sites showed remarkable pliability. The skin at these SASS-grafted sites could be pinched and readily regained its initial shape (Fig. 5g) without inducing blistering. When grafted on joints, SASSs did not restrict movement (Fig. 7g). Objective measurements of SASS, AG and uninjured skin sites located on equivalent anatomic regions were performed as described (Nedelec et al., 2008; Nedelec et al., 2016) to determine the skin thickness and elasticity (Table 2). No statistical difference in elasticity or thickness was found between the SASS and AG or the SASS and uninjured skin, which may be attributed to the small sample sizes.

Colour and aesthetic aspects of the SASSs after grafting

Some redness and skin elevation were observed in the SASSs, but not within the skin substitute (Fig. 5e and
Fig. 6d, black arrows). However, this hypertrophy and erythema disappeared over time (Fig. $5 \mathrm{f}$ and Fig. 6e). In some cases, the development of more visible scars associated with surface irregularities and increase thickness was noted with the meshed AGs

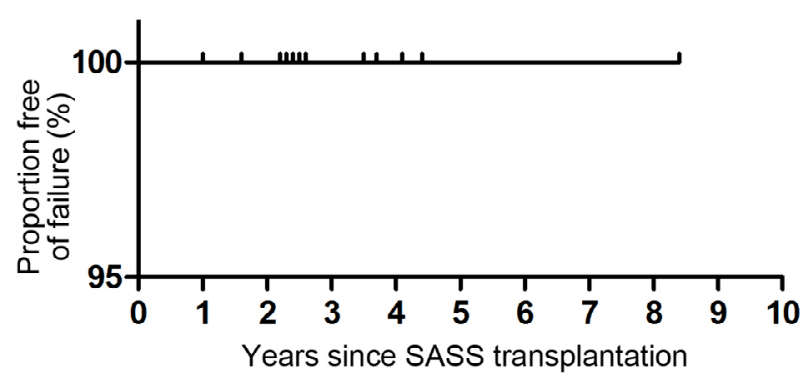

Fig. 4. Kaplan-Meier estimate of SASS epithelial cell survival after grafting based on the clinical observations at the last follow-up visit at the hospital.
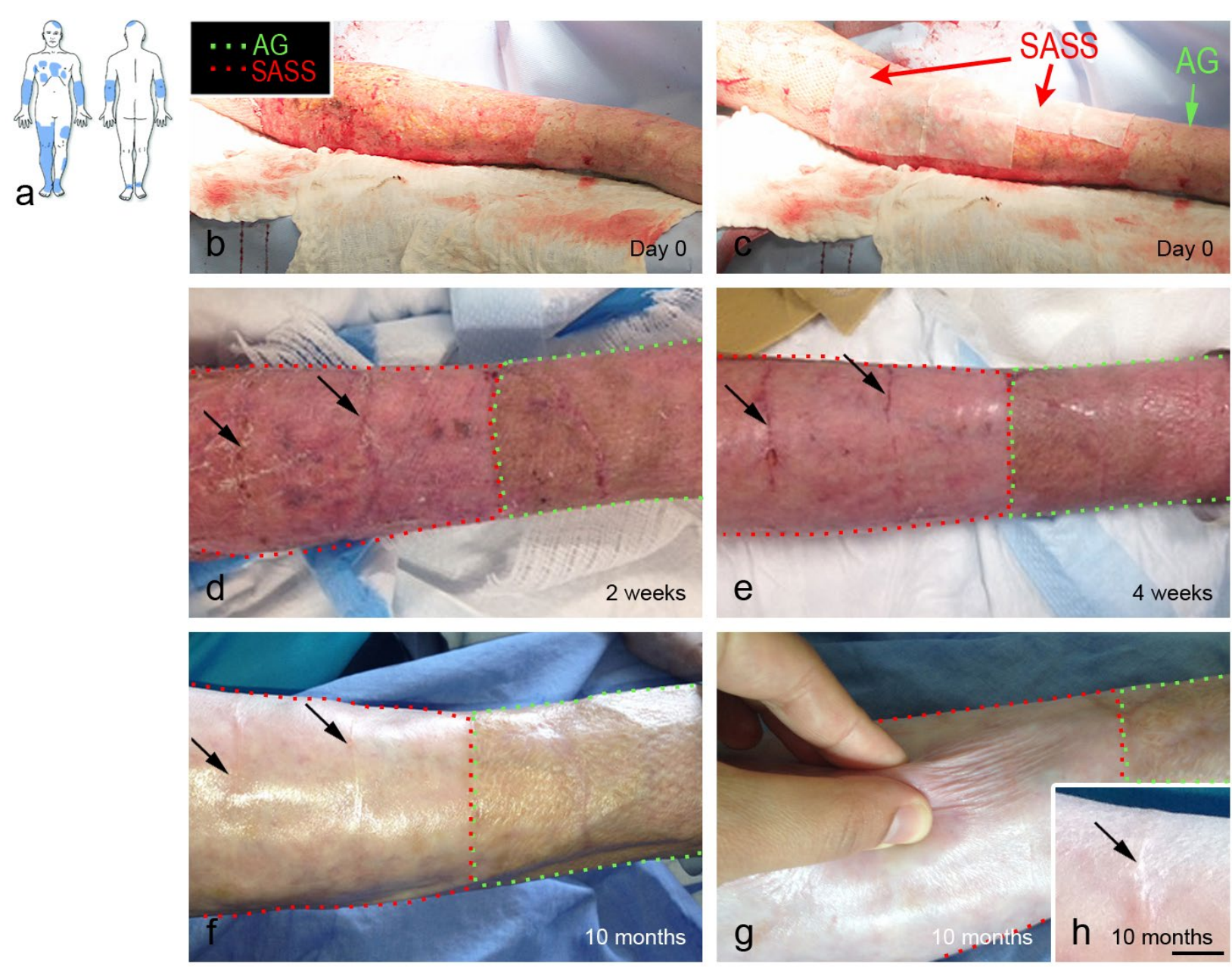

Fig. 5. Clinical observations of an adult patient severely burned over $80 \%$ of his TBSA who received SASSs over $24 \%$ of his burn area. (a) Grafted SASS' location is coloured in blue. (b) Full-thickness burn debridement over the left forearm prior to SASS application. Images of SASSs applied on (c) the wound bed, after (d) 2 weeks, (e) 4 weeks and (f-h) 10 months. (e-f) Some redness observed between SASSs during the early post-grafting period disappeared over time (black arrows). (f) Note the absence of a meshed pattern for SASSs (left side) as compared to AGs (right side). SASSs were hypopigmented, soft, pliable, supple and elastic as shown in g. (h) Magnification (scale bar: $5 \mathrm{~mm}$ ) of a junction between two juxtaposed SASSs. Grafted SASSs and AGs are delimited by red and green dotted lines, respectively. 
(Fig. 8c), while SASS-grafted sites appeared smooth and had a uniform thickness (Fig. 8b,d,e). Fig. 5f shows the macroscopic appearance of a SASS-grafted site next to a meshed AG site where the mesh pattern remained visible (Fig. 5f).

Erythema (vascularity) and melanin content (pigmentation) of the SASS, AG and uninjured skin sites located on equivalent anatomic regions were quantified. Even if obvious differences in colour between SASS and uninjured skin sites were visible in some patients, especially in more darkly pigmented patients (Fig. 7), no statistically significant difference was detected between the groups, probably due to the high variability of this parameter, the fact that sites most representative of the overall outcome were selected and the small number of cases (Table 2). However, SASS-grafted sites tended to have a smaller melanin index as compared to AGs and uninjured skin.

In some instances, pigmented zones spontaneously appeared at the SASS-grafted sites and enlarged over time (Fig. 6 and Fig. 7, asterisks). In one patient with a Fitzpatrick IV skin phototype (Fig. 7), the number of pigmented spots was larger when compared to other Caucasian patients (Fig. 5-9).

Another interesting characteristic was the capability of the grafted SASSs to expand when the patient grew or gained weight. Notably, three young patients (10, 13 and 15 years old) were treated with SASSs before puberty. After their growth spurt, these children did not report any sensation of skin tightness or having the slightest limitation of movement at the SASS-grafted sites. In two of these patients, surgical releases to address contractures were conducted at sites grafted with AGs, but it was not required for sites grafted with SASSs.

\section{Discussion}

The clinical outcomes of the SASSs used as permanent grafts for the acute treatment of full-thickness skin burns were presented. The handling and grafting of the SASSs were similar to AGs and no significant pitfalls or problems were encountered. SASSs showed excellent graft-take and stability as well as an aesthetic outcome equivalent to autografting. No loss of epithelium or skin breakdown was observed during the first year post-intervention or reported thereafter, indicating that SASSs supported the preservation of functional epithelial stem cells. Erythema, elasticity and thickness of skin suggested comparable results between SASSs, AGs and uninjured skin with objective measures. Further studies with uniform data collection from a larger number of subjects will be required to strengthen these conclusions and gain statistical power.

To increase the coverage, AGs can be meshed, with variable expansion ratios. However, concerns remain about the long-term effects that meshing
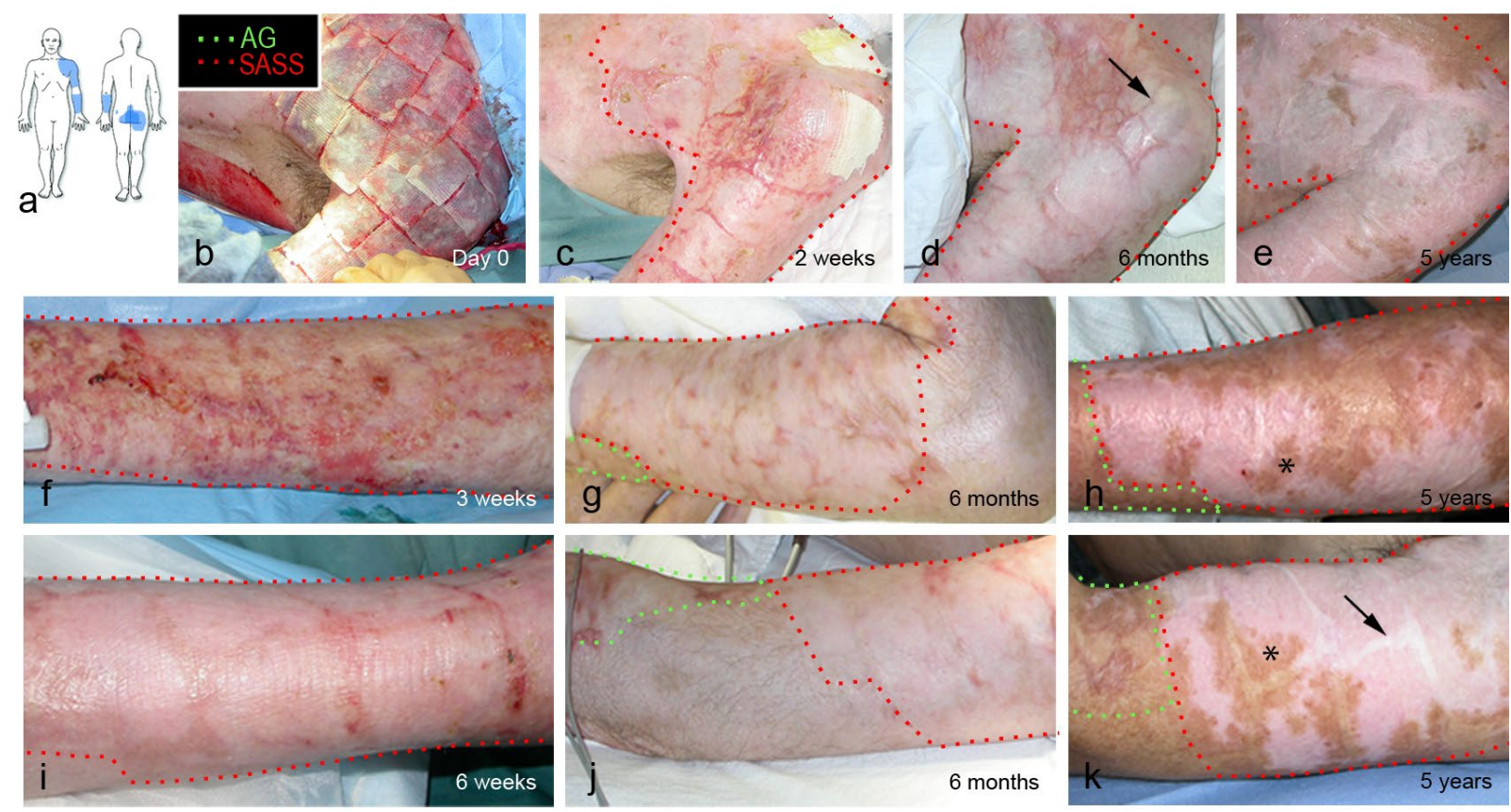

Fig. 6. Clinical observations of an adult patient severely burned over $85 \%$ of his TBSA who received SASSs over $13 \%$ of his burn area. (a) Grafted SASS' location is coloured in blue. (b-k) Images of SASSs grafted on (b-e) the shoulder, (f-h) the forearm and (i-k) the arm of the patient (b) immediately after grafting, after (c) 2 weeks, (f) 3 weeks, (i) 6 weeks, (d,g,j) 6 months and (e,h,k) 5 years. (d) Some skin redness and elevation between SASSs were observed during the first weeks (arrow), but not within the SASS, eventually disappeared over time. $(\mathbf{h}, \mathbf{k})$ Pigmented regions within the SASS appeared over time in this patient (asterisks). Arrows point to a junction between two juxtaposed SASSs. Grafted SASSs and AGs are delimited by red and green dotted lines, respectively. 
has on the range of motion, particularly due to scar contractures. Furthermore, aesthetic consequences are undeniable with meshed AGs since the mesh pattern is quite apparent and permanent (Herd et al., 1987). Hypertrophic scars and contractures are the main cutaneous complication in patients surviving severe burn injuries. They are responsible for severe functional limitations and unfavourable appearances (Finnerty et al., 2016). These limitations may cause long-term disabilities and challenges for return to work and result in high healthcare and loss of productivity costs. Thus, the high-quality outcomes documented in this study after SASS grafting potentially could contribute to a reduction of morbidity and an increase in the quality of life for burn survivors. Given the small size of this clinical sample, more studies will be needed to better evaluate skin characteristics and benefits of SASSs in terms of clinical outcomes, quality of life and cost-effectiveness. However, the absence of physical limitations, as well as the capability to mature and grow during a child's development were essential factors observed in the current study that demonstrated the potential benefits of this innovative technology.

The cohort of the present study consisted of patients ranging from 10 to 63 years of age. Using the revised Baux score, the average predicted mortality of this population was $52.7 \%$. The 2016 National Burn Repository (NBR) report summarises and compares outcomes for burn victims submitted by 96 American, 4 Canadian, 2 Swedish and 1 Swiss burn centres for the period between 2006 and 2015 . According to this report, the published mortality rate for a comparable population matched with the Baux score was $53.7 \%$. Using other autologous engineered skin substitutes, recent studies indicate a reduction of mortality in severely burned paediatric patients when skin substitutes are used (Boyce et al., 2017). Further studies with larger sample sizes are needed to confirm a reduction of mortality associated with the application of a SASSs.

Hirsch et al. (2017) describe a successful graft over $80 \%$ of a patient TBSA with transgenic keratinocytes cultured according to a method similar to the epithelial cell culture method described in this study. The transgene is expressed during the 21-month follow-up. Thus, this trial succeeds in definitively correcting epidermolysis bullosa and undoubtedly shows the long-term therapeutic effect of living CAE substitutes when cultured under appropriate conditions. In the present study, it was not possible to prove that the epidermis present in the SASS-grafted sites was generated from the construct itself because cultured keratinocytes composing the SASSs were not genetically tagged. However, it was unlikely that the epidermis originated from sources other than the SASSs due to the extent and depth of the burn wounds.

The main limitation of the autologous SASSs was the production time. Improved methods that require 2 weeks for cell amplification and 31 to $45 \mathrm{~d}$ to produce the SASSs are described (Larouche et al., 2016). Amongst bilayered skin substitutes that are tested on humans to treat full-thickness burn wounds, production time lines vary between 9 to 19 days (Boyce et al., 2006; Llames et al., 2004). However, regardless of the tissue-engineered method, the primary culture
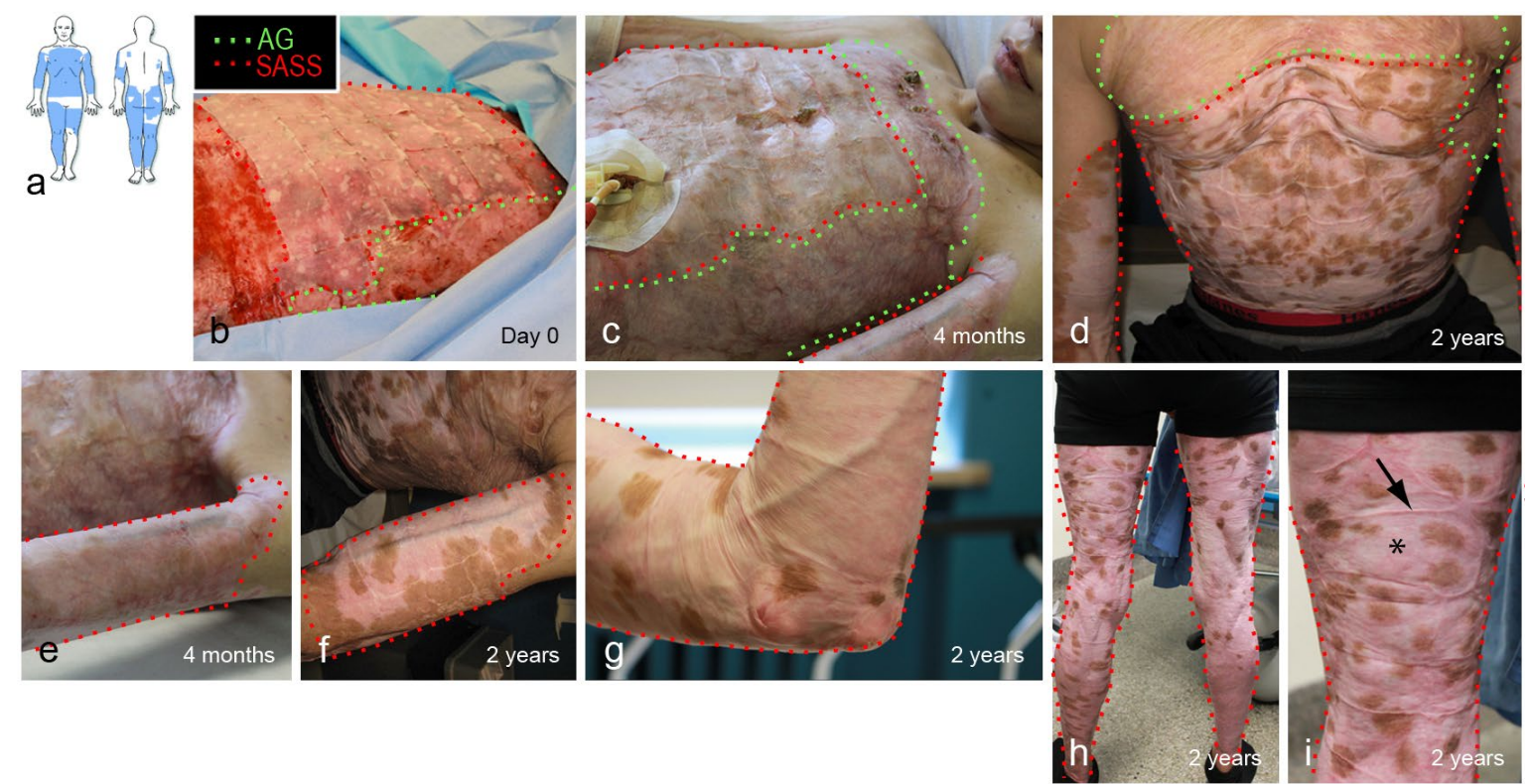

Fig. 7. Clinical observations of a teenager patient severely burned over $75 \%$ of his TBSA who received SASSs over $49 \%$ of his burn area. (a) Grafted SASS' location is coloured in blue. (b-i) Images of SASSs grafted on (b-d) the chest, $(\mathbf{e}-\mathbf{g})$ the arm and $(\mathbf{h}, \mathbf{i})$ the leg of the patient after $(\mathbf{c}, \mathbf{e}) 4$ months and $(\mathbf{d}, \mathbf{f}-\mathbf{i}) 2$ years. (d,f-i) Large number of pigmented spots 2 years after SASS grafting (asterisk) in this patient with a Fitzpatrick skin phototype IV. The arrow points to a junction between two juxtaposed SASSs, which remained discernible after 2 years. Grafted SASSs and AGs are delimited by red and green dotted lines, respectively. 
Table 2. Skin characteristic comparison between SASS, AG and uninjured skin. S, uninjured skin. ${ }^{1}$ Calculated with the Wilcoxon signed-rank test comparing mean differences.

\begin{tabular}{|c|c|c|c|c|c|c|c|}
\hline & $n$ & Mean & SD & Median & Min & Max & $p^{1}$ \\
\hline \multicolumn{8}{|c|}{ Thickness (mm) } \\
\hline SASS & 7 & 1.8 & 0.6 & 1.9 & 1.0 & 2.5 & \\
\hline AG & 7 & 1.8 & 0.5 & 1.9 & 1.2 & 2.5 & \\
\hline$S$ & 3 & 1.3 & 0.0 & 1.1 & 1.1 & 1.7 & \\
\hline SASS-AG & 7 & 0.0 & 0.3 & & & & 0.578 \\
\hline SASS-S & 3 & 0.7 & 0.8 & & & & 0.5 \\
\hline \multicolumn{8}{|c|}{ Maximum deformation (mm) } \\
\hline SASS & 7 & 0.80 & 0.37 & 0.73 & 0.32 & 1.40 & \\
\hline $\mathrm{AG}$ & 7 & 0.93 & 0.40 & 0.87 & 0.29 & 1.44 & \\
\hline$S$ & 3 & 1.18 & 0.82 & 1.58 & 0.23 & 1.73 & \\
\hline SASS-AG & 7 & -0.16 & 0.34 & & & & 0.578 \\
\hline SASS-S & 3 & -0.41 & 0.38 & & & & 0.5 \\
\hline \multicolumn{8}{|c|}{ Erythema (arbitrary unit) } \\
\hline SASS & 7 & 302 & 91 & 290 & 205 & 455 & \\
\hline $\mathrm{AG}$ & 7 & 322 & 63 & 291 & 275 & 441 & \\
\hline$S$ & 3 & 261 & 25 & 259 & 237 & 288 & \\
\hline SASS-AG & 7 & -15 & 57 & & & & 0.469 \\
\hline SASS-S & 3 & 53 & 63 & & & & 1 \\
\hline \multicolumn{8}{|c|}{ Melanin content (arbitrary unit) } \\
\hline SASS & 7 & 36 & 53 & 19 & 0 & 232 & \\
\hline AG & 7 & 101 & 83 & 78 & 27 & 275 & \\
\hline$S$ & 3 & 130 & 39 & 114 & 101 & 174 & \\
\hline SASS-AG & 7 & -73 & 92 & & & & 0.078 \\
\hline SASS-S & 3 & -99 & 95 & & & & 0.5 \\
\hline
\end{tabular}

steps of cell extraction and amplification depend on their capacity to proliferate in culture and delay the availability of skin substitutes (Boyce et al., 2006; Llames et al., 2004). ESS, the skin substitute tested in the larger number of patients, require a large number of fibroblasts to start the production (Boyce et al., 2006). Therefore, in the two main studies that use ESS to treat severely burned patients, 24 to $56 \mathrm{~d}$ are required from the skin harvest to the first graft (Boyce et al., 2006; Boyce et al., 2017). Shortest production time is reported in studies using artificial dermis based on human plasma (Gomez et al., 2011; Llames et al., 2006). With this method, the total production delay after harvesting skin biopsies ranges from 24 to $26 \mathrm{~d}$. However, the plasma-derived matrix does not provide mechanical properties conferred by collagen fibres. Autologous SASSs, with their high graft-take and mechanical properties satisfying surgeons' requirements are currently suited for severely burned patients who need longer acute care. The authors are actively developing production strategies to reduce the fabrication time of the SASSs to allow for earlier grafting (Beaudoin Cloutier et al., 2015).

Given that less than $10 \mathrm{~cm}^{2}$ of healthy donor skin is required to produce several thousand $\mathrm{cm}^{2}$ of autologous SASSs (up to $6925 \mathrm{~cm}^{2}$ in the present study), this technology could potentially solve the problem of insufficient donor site availability. Length of stay is the usual indicator for hospital administrators to evaluate direct costs related to hospitalisation, regardless of the final outcomes. However, it is more difficult to evaluate indirect costs associated with the loss of working days, disability and reduced quality of life that severely burned patients have to face. Considering the good clinical outcome of grafted SASSs and the possible reduction in production time in the future, the cost-benefit ratio of this technology may end up being positive, but further research is required to quantify these potential benefits.

The principal adverse events linked to the SASS application were pigmentation flaws (complete absence of pigmentation initially or development of progressive pigmented spots), skin dryness, lack of sweat and transient erythema. Hypopigmentation or scattered pigmented spots are also observed in ESS or CAE after grafting and result from the dilution of melanocytes that survive but replicate more slowly than keratinocytes and poorly recover from cryopreservation (Boyce et al., 2006; Compton et al., 1998; Harriger et al., 1995; Medalie et al., 1998). As melanocyte growth can potentially induce metaplasia or dysplasia, follow-up of the patients was performed and is still ongoing. Although, it should be noted that injection of cultured melanocytes in athymic mice does not induce any cancer development (Boyce et al., 2015). Skin dryness and lack of sweat at SASSgrafted sites were expected since no appendages are 

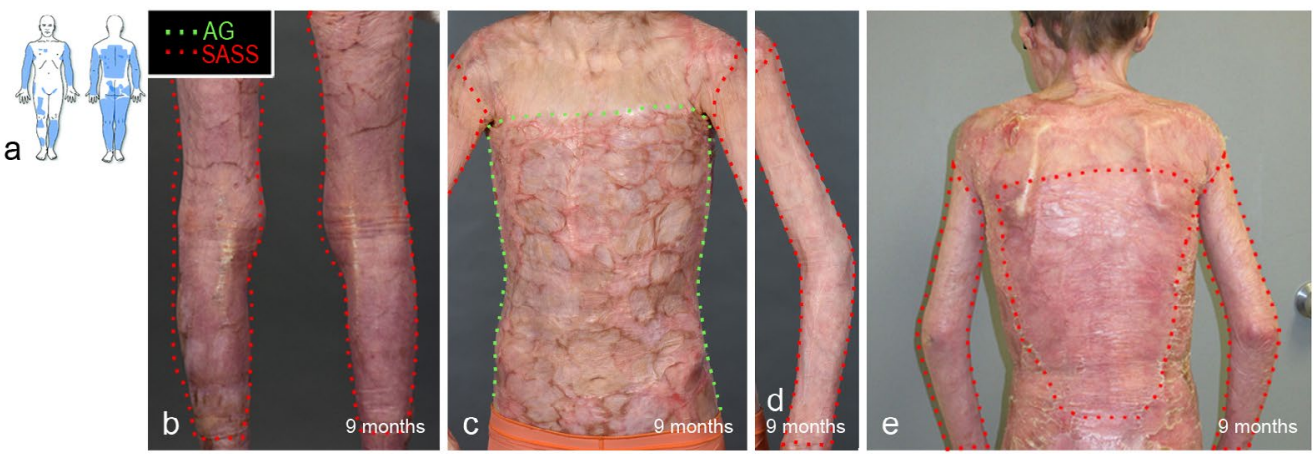

Fig. 8. Clinical observations of a child patient severely burned over $92 \%$ of his TBSA who received SASSs over $53 \%$ of his burn area. (a) Grafted SASS' location is coloured in blue. (b,d,e) Images of SASSs grafted on (b) the legs, $(\mathbf{c}, \mathbf{d}, \mathbf{e})$ the arms and (e) the back of the patient after 9 months. (c) AGs grafted on the chest of the patient after 9 months. The SASSs provided homogeneous stable healing with less visible scars (e) when compared with the chest grafted with AGs (c). Grafted SASSs and AGs are delimited by red and green dotted lines, respectively.
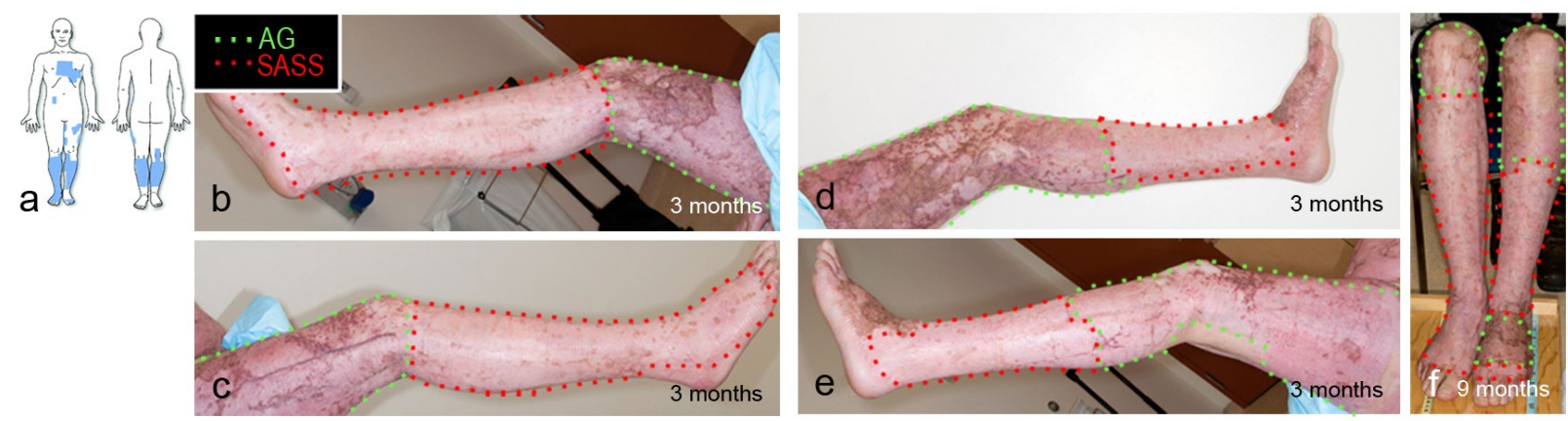

Fig. 9. Clinical observation of an adult patient severely burned over $90 \%$ of his TBSA who received SASSs over $18 \%$ of his burn area. (a) Grafted SASS' location is coloured in blue. (b-f) Images of SASSs grafted on the legs of the patient after (b-e) 3 and (f) 9 months. Grafted SASSs and AGs are delimited by red and green dotted lines, respectively.

present in SASSs to replace the sebaceous and sweat glands destroyed in full thickness burns. Finally, some patients experienced abnormal sensations (such as pruritus) at the site of their healed SASSs, which are common consequences associated with healed full-thickness burns (Malenfant et al., 1996).

\section{Conclusions}

This study presented an innovative technology in acute burn care and supports the use of SASSs as a valid therapeutic option in the treatment of fullthickness burn injuries covering more than $50 \%$ of the TBSA and requiring permanent coverage. These data indicated that stem cells were successfully engrafted within the SASSs ensuring the long-term epithelial self-renewal in all treated patients. The authors concluded that the SASS was an extremely promising skin substitute for permanent coverage of burn wounds given its simple utilisation, high graft-take rate, low infection rate, strong functional characteristics and its eventual capability of limiting hypertrophic scars and associated debilitating effects.
These characteristics rendered this substitute a clinically valuable choice for adults as for paediatric patients, notably due to its capability to expand with the child's growth. A randomised, paired-site comparison trial of SASSs and AGs for the treatment of full-thickness burns is currently underway.

\section{Acknowledgments}

We are grateful to the research professionals of the LOEX centre involved in the production of SASSs for the treatment of patients as well as to the staff of the burn units who provided care to the patients. The authors acknowledge all the plastic surgeons who provided their technical expertise in grafting and their clinical involvement in the follow-up of the SASSs. The authors would like to thank Dr Dong Hyun Kim (dermatologist) and Dr Steve Boyce for their expert advice. Funding: The Fondation des Pompiers du Québec pour les Grands Brûlés (FPQGB) (F.A.A, L.G., V.J.M. and B.N.), the Réseau de Thérapie Cellulaire et Tissulaire du FRQS (ThéCell) (F.A.A., L.G., B.M.K. and V.J.M.), the Canada Research Chair 
(Tier 1) on Stem Cells and Tissue Engineering (L.G.), the Research Chair on Tissue-Engineered Organs and Translational Medicine of the Fondation de l'Université Laval (L.G.), the Fondation du CHU de Québec-Université Laval, Canadian Institutes of Health Research (CIHR) grant MOP-14364 (F.A.A.), MOP-115093 (F.A.A. and V.J.M.), MOP-12087 and FDN-143213 (L.G.).

All authors contributed substantially to the acquisition, analysis or interpretation of the data, revised the manuscript, participated in drafting the article or revised it critically for important intellectual content, approved the final version to be published and agreed to be accountable for all aspects of the work. Additional responsibilities included the following: development of the SASS technology, conception and design of the work: F.A.A., L.G., D.L. and V.J.M.; ethical, legal and social implications: B.M.K. and M.T.N; histopathology: V.B; conception, design and measurement of clinical skin characteristics: J.K., J.L., V.J.M. and B.N.; patient treatment and follow-up: C.B.C., E.B., P.B., A.B., L.C.L., A.D., L.D., H.G. and I.P.; writing of the manuscript: D.L.; study supervision: F.A.A., L.G. and V.J.M.; co-senior authors: F.A.A., L.G. and V.J.M.

The authors declare no conflict of interest.

\section{References}

2016 National burn repository. Report of data from 2006-2015. Version 12.0. American burn association, Chicago.

Beaudoin Cloutier C, Guignard R, Bernard G, Gauvin R, Larouche D, Lavoie A, Lacroix D, Moulin VJ, Germain L, Auger FA (2015) Production of a bilayered self-assembled skin substitute using a tissue-engineered acellular dermal matrix. Tissue Eng Part C Methods 21: 1297-1305.

Bisson F, Rochefort E, Lavoie A, Larouche D, Zaniolo K, Simard-Bisson C, Damour O, Auger FA, Guerin SL, Germain L (2013) Irradiated human dermal fibroblasts are as efficient as mouse fibroblasts as a feeder layer to improve human epidermal cell culture lifespan. Int J Mol Sci 14: 4684-4704.

Black AF, Berthod F, L'Heureux N, Germain L, Auger FA (1998) In vitro reconstruction of a human capillary-like network in a tissue-engineered skin equivalent. FASEB J 12: 1331-1340.

Boa O, Cloutier CB, Genest H, Labbe R, Rodrigue B, Soucy J, Roy M, Arsenault F, Ospina CE, Dube N, Rochon MH, Larouche D, Moulin VJ, Germain L, Auger FA (2013) Prospective study on the treatment of lower-extremity chronic venous and mixed ulcers using tissue-engineered skin substitute made by the self-assembly approach. Adv Skin Wound Care 26: 400-409.

Boyce ST, Goretsky MJ, Greenhalgh DG, Kagan RJ, Rieman MT, Warden GD (1995a) Comparative assessment of cultured skin substitutes and native skin autograft for treatment of full-thickness burns. Ann Surg 222: 743-752.

Boyce ST, Kagan RJ, Greenhalgh DG, Warner P, Yakuboff KP, Palmieri T, Warden GD (2006) Cultured skin substitutes reduce requirements for harvesting of skin autograft for closure of excised, full-thickness burns. J Trauma 60: 821-829.

Boyce ST, Kagan RJ, Meyer NA, Yakuboff KP, Warden GD (1999) The 1999 clinical research award. Cultured skin substitutes combined with Integra Artificial Skin to replace native skin autograft and allograft for the closure of excised full-thickness burns. J Burn Care Rehabil 20: 453-461.

Boyce ST, Kagan RJ, Yakuboff KP, Meyer NA, Rieman MT, Greenhalgh DG, Warden GD (2002) Cultured skin substitutes reduce donor skin harvesting for closure of excised, full-thickness burns. Ann Surg 235: 269-279.

Boyce ST, Simpson PS, Rieman MT, Warner PM, Yakuboff KP, Bailey JK, Nelson JK, Fowler LA, Kagan RJ (2017) Randomized, paired-site comparison of autologous engineered skin substitutes and splitthickness skin graft for closure of extensive, fullthickness burns. J Burn Care Res 38: 61-70.

Boyce ST, Warden GD, Holder IA (1995b) Noncytotoxic combinations of topical antimicrobial agents for use with cultured skin substitutes. Antimicrob Agents Chemother 39: 1324-1328.

Boyce ST, Zimmerman RL, Supp DM (2015) Tumorigenicity testing in athymic mice of cultured human melanocytes for transplantation in engineered skin substitutes. Cell Transplant 24: 1423-1429.

Compton CC, Warland G, Kratz G (1998) Melanocytes in cultured epithelial grafts are depleted with serial subcultivation and cryopreservation: implications for clinical outcome. J Burn Care Rehabil 19: 330-336.

Dokter J, Meijs J, Oen IM, van Baar ME, van der Vlies CH, Boxma H (2014) External validation of the revised Baux score for the prediction of mortality in patients with acute burn injury. J Trauma Acute Care Surg 76: 840-845.

Finnerty CC, Jeschke MG, Branski LK, Barret JP, Dziewulski P, Herndon DN (2016) Hypertrophic scarring: the greatest unmet challenge after burn injury. Lancet 388: 1427-1436.

Fowler A, Dempsey A (1998) Split-thickness skin graft donor sites. J Wound Care 7: 399-402.

Gauvin R, Larouche D, Marcoux H, Guignard R, Auger FA, Germain L (2013) Minimal contraction for tissue-engineered skin substitutes when matured at the air-liquid interface. J Tissue Eng Regen Med 7: 452-460.

Golinski P, Menke H, Hofmann M, Valesky E, Butting M, Kippenberger S, Bereiter-Hahn J, Bernd A, Kaufmann R, Zoeller NN (2014) Development and characterization of an engraftable tissue-cultured skin autograft: alternative treatment for severe electrical injuries. Cells Tissues Organs 200: 227-239.

Gomez C, Galan JM, Torrero V, Ferreiro I, Perez D, Palao R, Martinez E, Llames S, Meana A, Holguin P 
(2011) Use of an autologous bioengineered composite skin in extensive burns: clinical and functional outcomes. A multicentric study. Burns 37: 580-589.

Harriger MD, Warden GD, Greenhalgh DG, Kagan RJ, Boyce ST (1995) Pigmentation and microanatomy of skin regenerated from composite grafts of cultured cells and biopolymers applied to full-thickness burn wounds. Transplantation 59: 702-707.

Herd AN, Hall PN, Widdowson P, Tanner NS (1987) Mesh graft - an 18 month follow-up. Burns Incl Therm Inj 13: 57-61.

Hirsch T, Rothoeft T, Teig N, Bauer JW, Pellegrini G, De Rosa L, Scaglione D, Reichelt J, Klausegger A, Kneisz D, Romano O, Secone Seconetti A, Contin R, Enzo E, Jurman I, Carulli S, Jacobsen F, Luecke T, Lehnhardt M, Fischer M, Kueckelhaus M, Quaglino D, Morgante M, Bicciato S, Bondanza S, De Luca M (2017) Regeneration of the entire human epidermis using transgenic stem cells. Nature 551: 327-332.

Kuroyanagi Y, Kenmochi M, Ishihara S, Takeda A, Shiraishi A, Ootake N, Uchinuma E, Torikai K, Shioya N (1993) A cultured skin substitute composed of fibroblasts and keratinocytes with a collagen matrix: preliminary results of clinical trials. Ann Plast Surg 31: 340-349; discussion 349-351.

Kyle MJ, Wallace AB (1950) Fluid replacement in burnt children. Br J Plast Surg 3: 194-204.

Larouche D, Cantin-Warren L, Desgagne M, Guignard R, Martel I, Ayoub A, Lavoie A, Gauvin R, Auger FA, Moulin VJ, Germain L (2016) Improved methods to produce tissue-engineered skin substitutes suitable for the permanent closure of full-thickness skin injuries. Biores Open Access 5: 320-329.

Larouche D, Hayward C, Cuffley K, Germain L (2005) Keratin 19 as a stem cell marker in vivo and in vitro. Methods Mol Biol 289: 103-110.

Larouche D, Paquet C, Fradette J, Carrier P, Auger FA, Germain L (2009) Regeneration of skin and cornea by tissue engineering. Methods Mol Biol 482: 233-256.

Lavoie A, Fugere C, Beauparlant A, Goyer B, Larouche D, Paquet C, Desgagne M, Sauve S, Robitaille H, Dunnwald M, Kim DH, Pouliot R, Fradette J, Germain L (2013) Human epithelial stem cells persist within tissue-engineered skin produced by the self-assembly approach. Tissue Eng Part A 19: 1023-1038.

Llames S, Garcia E, Garcia V, del Rio M, Larcher F, Jorcano JL, Lopez E, Holguin P, Miralles F, Otero J, Meana A (2006) Clinical results of an autologous engineered skin. Cell Tissue Bank 7: 47-53.

Llames SG, Del Rio M, Larcher F, Garcia E, Garcia M, Escamez MJ, Jorcano JL, Holguin P, Meana A (2004) Human plasma as a dermal scaffold for the generation of a completely autologous bioengineered skin. Transplantation 77: 350-355.

Lund CC, Browder NC (1944) The estimation of areas of burns. Surg Gynaecol Obstet 79: 352-358.

Malenfant A, Forget R, Papillon J, Amsel R, Frigon JY, Choiniere M (1996) Prevalence and characteristics of chronic sensory problems in burn patients. Pain 67: 493-500.
Medalie DA, Tompkins RG, Morgan JR (1998) Characterization of a composite tissue model that supports clonal growth of human melanocytes in vitro and in vivo. J Invest Dermatol 111: 810-816.

Michel M, L'Heureux N, Pouliot R, Xu W, Auger FA, Germain L (1999) Characterization of a new tissue-engineered human skin equivalent with hair. In Vitro Cell Dev Biol Anim 35: 318-326.

Nedelec B, Correa JA, Rachelska G, Armour A, LaSalle L (2008) Quantitative measurement of hypertrophic scar: intrarater reliability, sensitivity, and specificity. J Burn Care Res 29: 489-500.

Nedelec B, Forget NJ, Hurtubise T, Cimino S, de Muszka F, Legault A, Liu WL, de Oliveira A, Calva V, Correa JA (2016) Skin characteristics: normative data for elasticity, erythema, melanin, and thickness at 16 different anatomical locations. Skin Res Technol 22: 263-275.

Osler T, Glance LG, Hosmer DW (2010) Simplified estimates of the probability of death after burn injuries: extending and updating the baux score. J Trauma 68: 690-697.

Pouliot R, Larouche D, Auger FA, Juhasz J, Xu W, Li H, Germain L (2002) Reconstructed human skin produced in vitro and grafted on athymic mice. Transplantation 73: 1751-1757.

Sheridan RL, Morgan JR, Cusick JL, Petras LM, Lydon MM, Tompkins RG (2001) Initial experience with a composite autologous skin substitute. Burns 27: 421-424.

Takami Y, Yamaguchi R, Ono S, Hyakusoku H (2014) Clinical application and histological properties of autologous tissue-engineered skin equivalents using an acellular dermal matrix. J Nippon Med Sch 81: 356-366.

Voineskos SH, Ayeni OA, McKnight L, Thoma A (2009) Systematic review of skin graft donor-site dressings. Plast Reconstr Surg 124: 298-306.

Zuo KJ, Medina A, Tredget EE (2017) Important developments in burn care. Plast Reconstr Surg 139: $120 \mathrm{e}-138 \mathrm{e}$.

\section{Discussion with Reviewer}

Joel Fish: What are the limits of this current technology aside from the production time. What are the next steps for the technology and what are the costs for ordering $100 \mathrm{~cm}^{2}$ of SASS?

Authors: The production of SASSs currently requires the use of a feeder layer and xenogeneic products such as the foetal bovine serum for the preservation of epithelial stem cells in vitro. New culture conditions could be developed in the future.

SASSs are now in clinical trial in Canada (ClinicalTrials.gov Identifier: NCT02350205). The current cost is 3995 Canadian $\$ / 100 \mathrm{~cm}^{2}$.

Editor's note: The Scientific Editor responsible for this paper was Mauro Alini. 